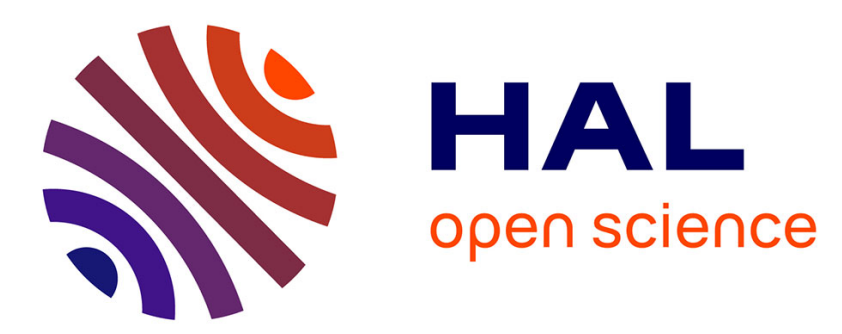

\title{
Se saisir du conseil de la vie lycéenne: des principes à l'exercice de la fonction de délégué
}

\author{
Valérie Becquet
}

\section{To cite this version:}

Valérie Becquet. Se saisir du conseil de la vie lycéenne: des principes à l'exercice de la fonction de délégué. Carrefours de l'éducation, 2009, Participation et éducation à la citoyenneté, 28, pp.65-80. $10.3917 /$ cdle.028.0065 . hal-03147410

\section{HAL Id: hal-03147410 https://hal.science/hal-03147410}

Submitted on 28 Feb 2021

HAL is a multi-disciplinary open access archive for the deposit and dissemination of scientific research documents, whether they are published or not. The documents may come from teaching and research institutions in France or abroad, or from public or private research centers.
L'archive ouverte pluridisciplinaire HAL, est destinée au dépôt et à la diffusion de documents scientifiques de niveau recherche, publiés ou non, émanant des établissements d'enseignement et de recherche français ou étrangers, des laboratoires publics ou privés. 


\title{
SE SAISIR DU CONSEIL DE LA VIE LYCÉENNE : DES PRINCIPES À L'EXERCICE DE LA FONCTION DE DÉLÉGUÉ
}

\author{
Valérie Becquet
}

Armand Colin | «Carrefours de l'éducation »

$2009 / 2 n^{\circ} 28 \mid$ pages 65 à 80

ISSN 1262-3490

Article disponible en ligne à l'adresse :

https://www.cairn.info/revue-carrefours-de-l-education-2009-2-page-65.htm

Distribution électronique Cairn.info pour Armand Colin.

(C) Armand Colin. Tous droits réservés pour tous pays.

La reproduction ou représentation de cet article, notamment par photocopie, n'est autorisée que dans les limites des conditions générales d'utilisation du site ou, le cas échéant, des conditions générales de la licence souscrite par votre établissement. Toute autre reproduction ou représentation, en tout ou partie, sous quelque forme et de quelque manière que ce soit, est interdite sauf accord préalable et écrit de l'éditeur, en dehors des cas prévus par la législation en vigueur en France. Il est précisé que son stockage dans une base de données est également interdit. 


\title{
Se saisir du conseil de la vie lycéenne: des principes à l'exercice de la fonction de délégué
}

\author{
$\Delta$ Valérie Becquet \\ becquetv@club-internet.fr
}

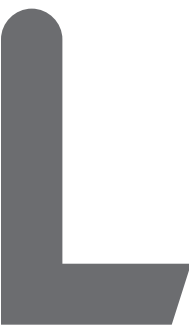

a création du conseil de la vie lycéenne fait suite à la consultation des lycéens initiée au printemps 1998 par Claude Allègre, alors ministre de l'Éducation nationale, de la Recherche et de la technologie. Dans un premier temps, un questionnaire est adressé aux lycéens et les conseils nationaux et académiques de la vie lycéenne sont saisis, puis, dans un second temps, un colloque est organisé en avril, dont les travaux débouchent sur

l'énonciation d'une série de principes et de propositions d'action. Parmi ceux-ci, le principe $n^{\circ} 42$ suggère la création dans chaque établissement d'un conseil de la vie lycéenne et en définit les contours. Ce conseil, dont la nouveauté est soulignée, aurait pour objectif « d'instaurer un dialogue plus efficace entre les lycéens et les autres membres de la communauté éducative sur toutes les propositions relatives à la vie et au travail scolaires ». Il s'inscrirait à ce titre dans une double logique: celle d'une relance de la démocratie lycéenne en référence à la défaillance des dispositifs de représentation des lycéens déjà existants et de l'application des droits obtenus en 1991 et, celle de l'amélioration de la participation des lycéens à la gestion de la vie de leur établissement. En revanche, à la différence d'autres démarches, ce nouveau conseil ne relèverait pas directement d'une démarche d'éducation à la citoyenneté. Après une phase d'expérimentation reposant sur le volontariat des établissements, cette instance est généralisée à l'ensemble des lycées publics en juillet 2000. À partir des résultats d'une enquête effectuée en 2002 auprès des membres du conseil de la vie lycéenne de neuf lycées publics, dont l'objet était d'analyser leur mise en place et leur fonctionnement, il s'agira de s'intéresser à l'accueil de cette nouvelle instance dans les établissements et, plus spécifiquement, à la manière dont les lycéens s'y sont engagés (Becquet, $2002^{1}$ ). Dans quelle

1. L'enquête s'est déroulée en 2002 auprès de neuf établissements répartis dans trois acadé- 
mesure se sont-ils saisis de cette opportunité? Quels sont les processus qui ont sous-tendu cet engagement? Pour tenter de répondre à ces questions, les objectifs et l'organisation de ce conseil sont rappelés. Puis, une analyse des modalités de réappropriation et d'intégration des lycéens en son sein est proposée.

\section{Principes et format du conseil de la vie lycéenne}

Dans les textes, le conseil de la vie lycéenne est présenté comme un espace permettant d'améliorer le dialogue à l'intérieur des établissements scolaires entre les lycéens et les autres membres de la communauté scolaire. Il articule deux principes, qui sont aussi deux approches du statut du lycéen: d'un côté, un principe politique où le lycéen est appréhendé comme un citoyen en formation, l'école devant lui permettre d'acquérir les compétences nécessaires à l'exercice de ce rôle et, de l'autre, un principe gestionnaire où le lycéen endosse la figure de l'usager et est habilité à faire valoir, dans un espace conçu à son intention, ses demandes et à donner son avis sur son cadre de travail.

Le principe politique repose sur une définition de l'établissement comme une « cité politique » qui confère à ses membres des droits et des obligations et, en particulier, le droit d'être représenté et d'être associé à la vie de l'établissement. Ce principe s'est progressivement affirmé et a sous-tendu la structuration de ce qu'il est désormais convenu d'appeler la « démocratie lycéenne » (Ballion, 1998). Il trouve son origine dans l'apparition de la notion de vie scolaire en 1945 et s'affirme à travers l'organisation progressive de la représentation des élèves (Soussan, 1988). La loi d'orientation de 1989, codifiée dans le Code de l'éducation, contribue largement à sa structuration. D'après ce texte, la formation du citoyen passe par la reconnaissance de droits et d'obligations aux lycéens et par la création dans les lycées publics d'un Conseil des délégués des élèves, laissant ainsi entendre que cette formation suppose un exercice immédiat de la citoyenneté. Ce conseil, qui ne sera mis en place qu'à la fin de l'année $1990^{2}$, systématise la rencontre entre les proviseurs, les délégués des élèves et d'autres acteurs de la communauté scolaire (Durand-Prinborgne, 1991; Roudet, 1994). Il est complété

mies aux caractéristiques différentes: un lycée d'enseignement général, trois lycées d'enseignement général et technologique, deux lycées professionnels et trois lycées polyvalents. 129 entretiens semi-directifs ont été réalisés auprès des membres des conseils de la vie lycéenne. Un guide d'entretien comportant une partie commune et une partie spécifique à chaque catégorie a été conçu afin d'appréhender les variations entre les catégories d'acteurs et entre les établissements. Depuis la réalisation de cette enquête, les élections des membres du conseil de la vie lycéenne ont eu lieu tous les deux ans, la dernière élection datant de la rentrée 2008. Bien que l'enquête ait été menée en 2002, les constats dressés au sujet du fonctionnement des conseils de la vie lycéenne ont été réitérés par la suite (voir par exemple le supplément de La Lettre de l'éducation n 454 du 7 juin 2004: Conseil de la vie lycéenne: un état des lieux et Guillaume et Verdon, 2007).

2. Décret n 90-978 du 31 octobre 1990 et Circulaire n 90-292 du 2 novembre 1990. 
en 1991 par la création du Conseil académique de la vie lycéenne et par celle, en 1995, du Conseil national de la vie lycéenne ${ }^{3}$.

Ainsi, au moment où est créé le conseil de la vie lycéenne, les lycéens bénéficient d'un système représentatif relativement complet puisqu'il couvre l'ensemble des niveaux décisionnels. Or, en 1998, cette « démocratie lycéenne » est présentée comme défaillante, les lycéens n'étant pas toujours reconnus comme des «partenaires politiques » (Rayou, 1998, p. 10) pertinents et rencontrant eux-mêmes des difficultés à endosser ce rôle au sein de leur établissement (Ballion, 1993 et 1998; Rayou, 1998). Le rapport final de la consultation de 1998 y fait référence et souligne également les attentes des lycéens en la matière: «Les lycéens par l'intermédiaire des délégués du CAVL souhaitent une meilleure organisation de la participation des délégués à la vie de l'établissement. Ils regrettent que l'élection de ceux-ci ne soit pas toujours prise au sérieux, préparée et utilisée dans la perspective d'une véritable formation à la démocratie ${ }^{4}$. ». Le rapport insiste également sur la nécessité de favoriser la concertation au sein des établissements et de permettre l'apprentissage du débat démocratique. C'est en référence à ces constats et à ces attentes que l'idée de mettre en place un conseil de la vie lycéenne est formulée et entérinée quelques mois plus tard.

La note de service ministérielle, intitulée Réforme des lycées: pour une participation accrue des élèves à la vie lycéenne ${ }^{5}$ invite les responsables des instances de participation à les « faire vivre pleinement ». Son contenu est repris par Claude Allègre au moment de la présentation à l'Assemblée nationale, suite au mouvement lycéen de l'automne 1998, du Plan d'action pour l'avenir des lycées. La partie consacrée à la démocratie lycéenne (Faire avancer la démocratie lycéenne) met en évidence que les textes de 1991 ne sont pas appliqués. Un renforcement de la participation des lycéens à tous les organes décisionnels et consultatifs est souhaité. Le conseil de la vie lycéenne est alors décrit comme une innovation permettant d'atteindre un tel objectif. Pourtant, si sa création apparaît en premier lieu relever d'un objectif de revitalisation de la démocratie lycéenne, elle est également sous-tendue par un autre principe qui, s'il n'est pas directement affirmé, se repère dans les discours et dans la manière dont est organisée cette nouvelle instance. Le principe gestionnaire postule l'éventuelle contribution de cette instance à l'administration des établissements scolaires en appréhendant les lycéens non plus comme des citoyens en formation mais comme des usagers. Il est moins mis en avant que le précédent pour des raisons contextuelles. Il s'agit avant tout de valoriser l'idée que la création du conseil témoigne que la demande des lycéens d'être davantage écoutés a été entendue et, par là même, de gommer un éventuel soupçon d'instrumentalisation des lycéens. De ce fait, les discours insistent peu sur cet objectif

3. Décret n 91-916 du 16 septembre 1991 et décret n 95-1293 du 18 décembre 1995. La création du Conseil national de la vie lycéenne a été annoncée dans le cadre du Nouveau contrat pour l'école. 4. Consultation des lycées. Rapport final du Comité d’organisation. Document ronéotypé. 1998. p. 13.

5. Réforme des lycées: pour une participation accrue des élèves à la vie lycéenne. Bulletin officiel, n 37, Ministère de l'Education nationale. 8 octobre 1998. 
ou alors, le formulent de telle manière que les lycéens soient présentés comme les principaux bénéficiaires de cette instance.

Or, à l'instar d'autres démarches participatives (Bacqué et al, 2005; Callon et al, 2001 ; François et Neveu, 1999), la création de ce conseil offre aux responsables des établissements scolaires la possibilité d'appréhender différemment leur action en direction des lycéens. L'amélioration du dialogue constitue un outil de management public permettant d'un côté, de mieux connaître les attentes et les difficultés des usagers et, de l'autre, de renforcer l'acceptabilité des décisions et, éventuellement, de prévenir des formes de contestation. Un tel objectif prend tout son sens au regard de l'évolution, depuis le milieu des années 1980, de l'administration des établissements scolaires et de la fonction de chef d'établissement (Barrère, 2006; Cousin, 1993; Dutercq, 2000). De ce fait, l'expérience des lycéens est appréhendée comme un usage de l'établissement qui peut être discuté au sein du conseil de la vie lycéenne. Les principes politique et gestionnaire ne sopposent pas mais constituent deux traductions différentes du dialogue qu'il est censé permettre. Il est clair que le principe politique apparaît plus légitime que le principe gestionnaire car il positionne, au moins formellement, le conseil du côté des revendications des lycéens et s'inscrit dans la continuité des discours sur les valeurs et les objectifs de l'école.

Afin de mettre en œuvre ces principes, plusieurs changements ont été apportés à l'organisation du conseil des délégués des élèves alors en place. Pour mémoire, ce conseil réunissait l'ensemble des délégués de classe, était présidé par le proviseur de l'établissement assisté du conseiller principal d'éducation et, élisait en son sein un bureau ${ }^{6}$. Ainsi, les échanges avaient principalement lieu entre les élèves, le proviseur, voire le proviseur adjoint, et le conseiller principal d'éducation. Étaient exclus de cette relation les autres membres de la communauté scolaire et, en particulier les enseignants. Autre caractéristique, l'effectif de l'assemblée variait en fonction du nombre de classes dans l'établissement, ce qui n'était pas sans conséquences sur la possibilité d'y débattre. Une telle configuration faisait ressembler ce conseil à une classe, seules les fonctions des participants changeant. En dehors des attributions qui restent identiques, le conseil de la vie lycéenne apporte plusieurs modifications à cette organisation de la relation. Premièrement, la composition de l'instance est nettement plus complète ${ }^{7}$. L'entrée des enseignants, des personnels ATOSS et des parents d'élèves fait qu'elle accueille désormais les différents acteurs de l'établissement et s'ouvre sur l'extérieur. Deuxièmement, le nombre de participants est réduit : dix lycéens et dix représentants des autres catégories siègent. Le proviseur reste le président mais un vice-président lycéen est élu. Troisièmement,

6. Décret n 90-978 du 31 octobre 1990 modifiant le décret n 85-924 du 30 août 1985 relatif aux établissements publics locaux d'enseignement.

7. Le conseil de la vie lycéenne est composé de sept lycéens élus pour deux ans, trois délégués des élèves élus pour un an, cinq enseignants ou personnels d'éducation, trois représentants des personnels administratifs, sociaux et de santé, technique, ouvriers et de service et deux représentants des parents d'élèves. 
les délégués ne sont pas seulement des délégués de classe. Sept sur dix sont élus par l'ensemble des élèves de l'établissement. Quatrièmement, les lycéens et les adultes n'ont pas le même pouvoir à l'intérieur de l'instance. Ainsi, si un vote doit avoir lieu pour décider d'une proposition ou de la formulation d'un vœu, seuls les lycéens peuvent prendre part au vote, les autres membres n'ayant qu'une voix consultative. Pour finir, la création du conseil de la vie lycéenne débouche sur, dans un premier temps, la transformation du conseil des délégués des élèves en conférence des délégués des élèves, celle-ci perdant une partie de ses prérogatives puis, dans un second temps, en 2004, à la demande des élus lycéens du conseil national de la vie lycéenne, sur la transformation de cette conférence en assemblée générale des délégués ${ }^{8}$.

Ainsi, le conseil de la vie lycéenne comporte des caractéristiques qui modifient très nettement les conditions du dialogue au sein de l'établissement ${ }^{9}$. Il se situe en fait à la croisée de deux modèles politiques - représentatif et participatif — et d'outils déjà existants. D'une part, sa composition est proche de celle du conseil d'administration même s'il n'a pas de pouvoir décisionnel. D’autre part, est maintenue une modalité traditionnelle de représentation des élèves, reprenant la figure du délégué de classe, mais sur un champ de compétence plus large. Pour finir, se constate un renforcement du modèle participatif c'est-à-dire valorisant la discussion entre différentes catégories d'acteurs pour traiter du fonctionnement des établissements et, en particulier des questions de vie lycéenne. De telles caractéristiques n'ont pas été sans conséquences sur la manière dont cette instance a été accueillie et investie.

\section{Une opportunité jugée inégalement crédible}

Afin d'organiser le scrutin du mois d'octobre 2000, la circulaire ministérielle du mois de juillet 2000 précisait que l'information des lycéens devait avoir lieu au cours du mois de septembre afin qu'ils aient le temps de se porter candidat et de faire campagne auprès de leurs camarades. L'organisation d'une élection au sein de l'établissement, préalable à l'installation de cette instance, venait s'ajouter à l'ensemble des tâches de la rentrée des proviseurs et des conseillers principaux d'éducation et des professeurs principaux. Il en a résulté une information inégale d'un établissement à l'autre. Ces variations tenaient soit à des problèmes de calendrier, le délai octroyé étant jugé insuffisant au regard d'un agenda de rentrée déjà chargé, soit à des problèmes pratiques, la taille de l'établissement ne permettant pas d'informer tous les élèves, soit à des problèmes d'expertise, les personnels ne parvenant pas toujours à présenter l'intérêt et l'utilité de cette nouvelle instance,

8. Pour une présentation actualisée des instances de la vie lycéenne: Circulaire n 2008-114 du 29 août 2008 relative à la composition et au fonctionnement des instances de la vie lycéenne.

9. Sur la question des effets de l'organisation d'un espace de discussion sur l'échange, voir, entre autres, Callon, Lascoumes et Barthe, 2001. 
soit à des problèmes de division du travail, la tâche d'informer les élèves pouvant tout autant relever du professeur principal, du conseiller principal d'éducation ou du chef d'établissement.

L'émergence de ces problèmes et, par là même, leur résolution, était également fortement liée à la conviction des acteurs chargés de contribuer à la mise en place de ce conseil (Becquet, 2005a). Ainsi, dans les établissements où les proviseurs considéraient cette mesure comme pertinente, la mobilisation a nettement été plus forte quels que soient la taille et le type d'établissement. Par exemple, dans le cas des lycées polyvalents, dont les effectifs étaient compris entre 1100 et 2200 élèves, ce qui pouvait nuire à l'information et à la mobilisation des élèves, l'engagement des proviseurs a différé en référence à leur appréhension du dispositif. Dans les deux lycées professionnels et dans un lycée d'enseignement général et technologique, les proviseurs et leurs adjoints se sont personnellement investis pour informer les lycéens en créant des supports et en intervenant dans les classes. Leur intérêt s'explique alors par leur volonté de développer une vie dans leur établissement et est également lié à l'existence d'un internat en leur sein. Dans les autres, ils ont chargé les conseillers principaux d'éducation ou les professeurs principaux de présenter le conseil aux élèves et de les inviter à se porter candidats. Ces différences entre les établissements se retrouvent dans les propos des lycéens qui, soit ne se souviennent plus de la manière dont ils ont été informés, soit jugent parcellaires les informations reçues. Les lycéens n'avaient d'ailleurs pas toujours compris ce qu'était ce conseil, en particulier ses objectifs et son fonctionnement, ce qui ne leur a pas nécessairement donné envie de se présenter aux élections et, par la suite d'aller voter.

De ce fait, le conseil de la vie lycéenne, s'il était perçu comme une nouvelle instance, ne bénéficiait pas auprès des lycéens d'un fort capital de sympathie, ces derniers ne le percevant pas toujours comme une réelle opportunité. Si la conviction des acteurs, proviseurs, comme conseillers principaux d'éducation et professeurs principaux et, les problèmes repérés ont pesé sur la manière dont ce conseil a été présenté aux lycéens et, par conséquent, sur leur appropriation de cette nouveauté, leur jugement s'est également construit en référence à leur expérience des dispositifs déjà existants. Ils ont ainsi évalué les objectifs affichés représenter les lycéens et donner leur avis sur la vie de l'établissement -, à l'aune d'une part, de leur expérience lycéenne et, d'autre part, de la possibilité d'exercer leur mandat au sein du conseil de classe, du conseil des délégués des élèves et du conseil d'administration et de la capacité de leurs interlocuteurs à les écouter. Au sujet de la première, il est fréquent d'insister sur sa variation en fonction des milieux sociaux d'appartenance et des caractéristiques des établissements et de rappeler l'accroissement de logiques de distanciation à l'égard de l'institution scolaire au profit, entre autres, de communautés de pairs qui en dépassent les frontières (Dubet, 1991 ; Rayou, 1998). Dans cette perspective, le lycée n'apparaît plus en tant que tel comme un lieu à vivre, justifiant un engagement dans des 
instances contribuant à l'amélioration de son organisation et de son quotidien, mais est devenu un lieu de passage. De ce fait, l'entrée dans une instance lycéenne n'est plus autant considérée comme socialement rentable au regard des sociabilités privilégiées. Cette première dimension est renforcée par une seconde qui renvoie davantage à l'exercice de la fonction de délégué de classe. Cette fonction semble faiblement valorisée par l'institution et jugée par les lycéens eux-mêmes comme difficile à exercer et inégalement efficace (Ballion, 1993 et 1998; Boumard, 1997 ; Merle, 2007; Rayou, 1998). Quant aux droits d'expression octroyés au début des années 1990, ils ne sont pas nécessairement appropriés. La définition qu'en ont les lycéens met plutôt en lumière une insistance sur leurs obligations aux dépens d'une maîtrise de leurs droits, en particulier de leurs droits politiques (Merle, 2001 et 2005; Robert, 1999). De plus, lorsqu'ils s'en saisissent, ils rencontrent parfois des difficultés qui interrogent la possibilité même de pleinement les exercer (Becquet, 2003).

L'appropriation de cette nouvelle instance par les lycéens a donc pris appui sur une inégale connaissance de ses spécificités et sur un jugement construit à partir d'une représentation ou d'une expérience de la possibilité d'intervenir dans l'enceinte scolaire. Ce n'était pas tant l'organisation du conseil de la vie lycéenne qui était remise en cause que la perception de son éventuelle efficacité au regard de l'existant. L'opinion des lycéens fait directement écho aux deux principes qui sous-tendent le conseil de la vie lycéenne. De manière paradoxale, la valorisation du principe politique dans les discours a une efficacité relative auprès des lycéens compte tenu de leur expérience de la représentation collégienne et lycéenne. Et quant au principe gestionnaire, plus discret, les lycéens se présentent soit comme des usagers distanciés, ne faisant que " passer » dans leur établissement, soit comme des usagers stratèges, préférant développer des démarches individuelles pour régler d'éventuelles difficultés. Ainsi, la conjugaison de deux principes et l'invitation au dialogue, si elles tentent de redéfinir les conditions de l'échange, s'inscrivent dans un continuum d'expériences qui pèse sur leur mise en œuvre. Les candidatures des lycéens en témoignent directement: ce sont ceux qui avaient une expérience positive de la fonction de délégation et ceux qui n'en avaient aucune, mais qui voyaient dans le conseil une manière d'agir plus attractive que le fait d'être délégué de classe, qui se sont plus fréquemment portés candidats.

L'analyse de l'exercice de la fonction de délégué au conseil de la vie lycéenne permet d'en appréhender d'autres aspects. En référence aux travaux s'intéressant au vécu des membres d'instances comme, par exemple, des conseils de jeunes ou des conseils de quartier (Bacqué et al., 2005; Becquet, 2005b; Blondiaux, 2000 ; Blondiaux et al., 1999), trois dimensions de cette expérience apparaissent particulièrement intéressantes à observer: la définition des objets de la discussion, la prise de parole des lycéens au cours des réunions et l'exercice du mandat de délégué au conseil de la vie lycéenne. Elles permettent de souligner les difficultés 
que rencontrent les lycéens à se saisir du conseil de la vie lycéenne et à établir un dialogue avec les autres membres.

\section{Des usages du conseil plus légitimes que d'autres}

Le champ de compétence du conseil de la vie lycéenne est relativement vaste, ce dernier pouvant traiter de l'ensemble des sujets qui relèvent de la vie et du travail scolaires ${ }^{10}$. Cette option signale aux lycéens que leur prise de parole n'est pas cantonnée à quelques sujets définis par l'institution mais qu'il est ouvert à leurs préoccupations. Cependant, cette ouverture ne facilite pas nécessairement la structuration d'un échange. En effet, les sujets dont il peut effectivement en réunion être débattu ne sont pas faciles à définir. La composition du conseil introduit une concurrence entre des préoccupations hétérogènes portées par des acteurs aux statuts différents et à l'inégale légitimité au sein de l'établissement.

Ainsi, alors que les lycéens sont invités à donner leur avis sur la vie de l'établissement au titre de l'usage qu'ils en font quotidiennement, ils se retrouvent limités dans cet exercice du fait de la présence d'autres catégories de personnels au conseil, en particulier les enseignants, ou du fait de leur évaluation des changements pouvant effectivement résulter de leur intervention. Par exemple, alors qu'ils passent principalement leur temps en classe, il est quasiment impossible d'aborder des questions touchant à cet espace. Les lycéens ne jugent pas pertinent d'intervenir sur le cas d'un professeur mais ils souhaiteraient faire part de leurs difficultés à concilier l'ensemble des demandes de leurs professeurs comme, par exemple, l'accumulation de contrôles au cours d'une même semaine. En parler leur semble d'autant plus important qu'ils ne parviennent pas nécessairement à le faire au cours des conseils de classe, se voyant rétorquer que ce n'est pas le lieu. Or, les proviseurs sont réticents à traiter de tels sujets, craignant que les enseignants présents se sentent mis en cause. Afin d'éviter les conflits, ils orientent les lycéens vers des débats plus généraux comme le projet d'établissement ou l'aide individualisée. Les enseignants de leur côté sont partagés à ce sujet, une partie s'opposant à toute discussion touchant à la classe, l'autre acceptant de l'avoir mais en leur nom propre. La situation est identique concernant les emplois du temps. Alors que les lycéens se plaignent d'emplois du temps trop chargés ou mal agencés, ils sont invités à engager une réflexion sur les rythmes scolaires. Or, ils ne se saisissent pas de cette opportunité car ils n'en perçoivent pas l'intérêt, l'échange n'aboutissant pas à un changement de leur situation. De

10. Domaines de compétence: les principes généraux de l'organisation des études, l'organisation du temps scolaire et l'élaboration du projet d'établissement ainsi que l'élaboration ou la modification du règlement; les modalités générales de l'organisation du travail personnel et du soutien des élèves; l'information liée à l'orientation et portant sur les études scolaires et universitaires, sur les carrières professionnelles; la santé, l'hygiène et la sécurité et l'aménagement des espaces destinées à la vie lycéenne; l'organisation des activités sportives, culturelles et préscolaires. Composition et attributions du conseil des délégués pour la vie lycéenne, Circulaire n²000-104 du 11 juillet 2000. 
plus, ils prennent progressivement conscience que les emplois du temps résultent de l'agencement de nombreuses contraintes et que les demandes des enseignants passent, entre autres, avant celles des lycéens. Rares sont les cas où, comme dans ce lycée d'enseignement général et technologique, les délégués ont obtenu la banalisation d'un créneau hebdomadaire d'une heure réservé à l'heure de vie de classe permettant, au passage, au conseil de se réunir et aux clubs de fonctionner. Dans le cas des emplois du temps, leur est généralement signifiée leur position de personnage secondaire dans une instance où ils sont censés être des usagers de premier plan. Seule la refonte des règlements intérieurs, souhaitée par les textes de juillet 2000, a, lorsque le proviseur a associé le conseil de la vie lycéenne, débouché sur un réel travail commun et a mis au passage en évidence la variété des perceptions des droits et des obligations entre leurs membres.

En fait, le cadre de vie est le sujet le plus abordé. Les élèves font remonter différentes demandes (variété des menus, garage à vélo, poubelles, etc.) et soulignent certains manques (papier hygiénique, savon, bancs, distributeurs de boissons, etc.). Leurs interventions se concentrent principalement sur l'amélioration de leur quotidien. Pourtant, alors que cette dimension entre dans les attributions du conseil, certains proviseurs portent un regard assez sévère sur le fait de n'aborder que des problèmes matériels. Ils reconnaissent la nécessité d'en parler mais oscillent entre étonnement et agacement. Ils jugent que les lycéens se comportent avant tout comme des consommateurs, privilégiant leur confort matériel sur des sujets plus sérieux. D'autres, en particulier les proviseurs de lycées professionnels et/ou dotés d'un internat, considèrent que cela peut leur permettre d'améliorer l'accueil des lycéens et de rendre l'établissement plus attractif. De leur côté, les personnels ATOSS ne sont pas étonnés que des questions d'ordre matériel soient traitées. Étant directement concernés, ils sont d'ailleurs généralement intéressés par ces sujets car ils touchent directement leur travail. Ils trouvent les élèves concrets et peuvent en profiter pour expliquer leur fonction ou certains choix, comme dans le cas de la restauration scolaire, ou pour leur faire part de leur mécontentement au sujet, par exemple, des mégots, des crachats et des détériorations.

Ainsi, les débats se focalisent sur quelques points ${ }^{11}$. Le champ de compétence, relativement large, est redéfini par les perceptions et les attentes respectives des participants. Par conséquent, d'un côté, les proviseurs constatent que les élèves n'abordent pas les sujets dont ils aimeraient qu'ils parlent ou aimeraient parler. De l'autre, les lycéens n'osent pas engager des discussions qu'ils souhaiteraient avoir afin d'éviter d'essuyer un refus ou de susciter une réaction de la part du proviseur, des enseignants, voire des conseillers principaux d'éducation au sujet

11. L'enquête réalisée par la DEPP en 2004-2005 sur la participation des lycéens souligne également que les propositions des élèves portent davantage sur « la qualité de vie au quotidien » que sur « les conditions d'enseignement à proprement parler » (Guillaume et Verdon, 2007). 
de la formation des délégués de classe. Le cadre de vie se présente alors comme un thème plus consensuel. Cette orientation des débats traduit bien la tension entre les principes politique et gestionnaire retenus et leur mise en œuvre. Le premier est valorisé à travers le souhait d'une montée en généralité qu'illustreraient par exemple des débats sur les rythmes scolaires qui concernent l'ensemble de la communauté scolaire tandis que le second apparaît moins légitime mais mobilise davantage les lycéens du fait des changements qu'il permet. À bien des égards, ils adoptent une posture « syndicale » visant à améliorer leurs conditions de travail (Merle, 2001). La construction d'un nécessaire compromis autour des objets discutables a également un impact direct sur la prise de parole des lycéens au cours des réunions, celle-ci étant également structurée par la manière dont les délégués parviennent à endosser leur rôle et à faire valoir leur position au sein de cette instance.

\section{Des délégués qui restent des élèves}

Les jugements des personnels et des parents d'élèves présents sont, à ce sujet, particulièrement éclairants. D’une manière générale, ils considèrent que les lycéens prennent inégalement la parole, voire qu'ils hésitent à intervenir. Malgré les efforts des proviseurs pour la susciter, l'expression lycéenne ne se caractérise pas par sa spontanéité et son importance. Cependant, l'impression générale qui se dégage des propos des adultes ne donne pas lieu aux mêmes interprétations. La majorité des enseignants fait preuve d'une certaine incompréhension face à la discrétion des lycéens. Selon eux, les lycéens ne devraient pas éprouver de difficultés à prendre la parole en réunion, voire devraient la prendre quasi naturellement. Deux raisons différentes sont évoquées pour étayer cette opinion. D’une part, le conseil de la vie lycéenne a été créé pour eux. Il leur permet de disposer d'une instance de plus où, par rapport au conseil de classe et au conseil d'administration, leur participation est censée être facilitée. D'autre part, les lycéens ne se gênent pas pour prendre la parole en classe. À ce sujet, les enseignants se réfèrent tout autant à la participation orale dans le cadre d'une demande scolaire qu'aux bavardages et aux chahuts. Aux yeux de ces enseignants, la situation d'échange offerte par le conseil apparaît par conséquent relativement idéale. Peu d'entre eux pointent les freins à l'expression des lycéens en réunion alors que c'est nettement plus souvent le cas des personnels et des parents. En effet, la plupart d'entre eux trouvent de multiples excuses aux hésitations des lycéens. D'un côté, ils ont une approche psychologique en se référant aux personnalités des élèves et à leurs effets sur l'attitude en réunion. De l'autre, ils considèrent que la présence du proviseur, des enseignants, voire de «tous ces adultes», ainsi que le côté officiel de la réunion, ont des effets non négligeables sur la participation des lycéens. La configuration de l'échange pourrait être source d'incertitudes et susciter certaines peurs. À bien des égards, la perception qu'ont ces acteurs de l'attitude des lycéens 
renvoie à leur propre expérience de la prise de parole. En effet, dans les entretiens, il est fréquent que les personnels de l'établissement et les parents d'élèves mettent en perspective leurs difficultés personnelles à intervenir, en particulier au conseil d'administration où ils siègent fréquemment, avec celles des élèves, adoptant une attitude particulièrement compréhensive à leur égard.

Le regard que portent les lycéens sur leur propre participation va dans le même sens que celui des adultes. Ils reconnaissent leur inégale contribution aux débats. Les plus discrets avouent se taire en réunion et les plus volubiles, qui ont souvent une primo expérience de la représentation des élèves, décrivent le silence de leurs condisciples. Ils justifient leur attitude de plusieurs manières, dont certaines sont identiques à celles soulignées par les personnels et les parents. La référence à la personnalité - timidité et discrétion - est toujours présente. Le silence s'explique également par le fait de se sentir ou non concerné par le sujet ou par celui d'avoir ou non un avis sur le sujet. Parler pour parler leur apparaît tout à fait superflu, beaucoup avouant de pas toujours avoir une opinion précise sur le sujet abordé. À ces deux aspects, s'ajoute une référence à l'instance elle-même. Premièrement, ils reconnaissent que la présence des adultes, en particulier celle du proviseur et des enseignants, influe sur leur prise de parole. Ils craignent les jugements, les sentences et les quolibets, ces sentiments se nourrissant de leur expérience de la classe, voire du conseil de classe (Ballion, 1998; Dubet, 1991; Merle, 2005 et 2007), et de leur perception de la fonction du proviseur, personne à laquelle il serait inopportun de s'opposer. Deuxièmement, bien que la situation soit différente, ils ont tendance à reproduire les comportements adoptés en classe, à savoir une écoute silencieuse et une participation orale marginale et stratégique (Barrère, 1997; Sembel, 2003). Troisièmement, ils ressentent une certaine incompétence linguistique et ont le sentiment de manquer d'informations sur les sujets abordés. Ainsi, la construction d'arguments valables pour peser sur le débat n'est pas toujours évidente. Il est clair que des variations existent d'un type de lycée à un autre. Les lycéens des filières professionnelles insistent davantage sur les problèmes de langue et leur sentiment d'être peu légitimes que les lycéens de l'enseignement général. De plus, entre les lycéens, le niveau scolaire ou l'expérience antérieure de la délégation pèsent sur leur participation. Ils ressentent pleinement le lien souvent établi au sujet des délégués de classe entre performance scolaire et légitimité politique (Barrère et Martucelli, 1998). La conjugaison de ces dimensions leur donne parfois l'impression que, malgré la bonne volonté des adultes, il n'est pas évident de participer aux échanges au cours des réunions. Les lycéens se sentent empêtrés dans des cadres, certains réels, d'autres fantasmés, qui les renvoient en permanence à leur position d'élève qu'ils vivent comme une situation d'infériorité. Par conséquent, alors que le fait d'endosser le rôle d'élève et l'exercice du métier d'élève se présentent, de part les exigences de conformité qu'ils posent, comme des conditions d'une intégration institutionnelle aboutie, voire d'une réussite scolaire (Perrenoud, 1994; Sirota, 1993), la participation au 
conseil de la vie lycéenne appelle un changement de posture que les délégués ne parviennent pas toujours à opérer. Ainsi, sa configuration, offrant un espace de dialogue a priori plus ouvert, rend finalement relativement plus délicate l'engagement des lycéens dans le débat.

Un tel processus se retrouve au niveau du travail des délégués des élèves entre les réunions. À ce sujet, les membres notent l'existence d'un décalage entre ce qui se passe en réunion et en dehors des réunions est mis en évidence. Ainsi le temps de discussion, même si la présence des lycéens et leur participation aux débats sont inégales, laisse penser que les délégués sont relativement intéressés par ce conseil. Or, les adultes ont le sentiment que les lycéens ne se côtoient pas entre les réunions et qu'ils éprouvent des difficultés à instaurer une collaboration. Ils constatent, d'une réunion à l'autre, le faible avancement des dossiers, voire le manque de préparation des réunions. Pourtant, plusieurs proviseurs estiment mettre à la disposition des lycéens les moyens nécessaires pour qu'ils se rencontrent. Certains bénéficient d'un local, d'autres peuvent demander une salle pour se réunir. Interrogés sur leur travail de délégué, les lycéens reconnaissent qu'ils se rencontrent peu entre les réunions plénières du conseil de la vie lycéenne. Certains se côtoient dans la cour et les couloirs alors que d'autres ne se voient jamais. Une collaboration n'est pas simple à construire, la discussion informelle étant parfois préférée même si elle ne permet pas nécessairement de travailler de concert sur les dossiers. C'est principalement du côté, d'une part, de la faible définition de la nature du mandat des élèves et, d'autre part, de l'organisation des établissements et de l'agenda scolaire qu'il convient de chercher des explications à cette situation.

Tout comme les modalités de fonctionnement du conseil de la vie lycéenne, en particulier les règles d'organisation du débat, ne sont pas définies à l'avance et se construisent au fil des réunions, le contenu du mandat de lycéens n'est pas précisé. Les lycéens ne savent pas nécessairement ce qu'ils sont censés faire en sus de leur présence aux réunions. De ce fait, ils calquent a priori leur travail sur une partie de celui du délégué de classe: recueillir des informations, assister aux réunions et restituer le contenu de la réunion. Or, ce schéma apparaît difficile à mettre en œuvre dans le cadre du conseil de la vie lycéenne du fait que cette instance s'adresse à l'ensemble des élèves d'un établissement. Ainsi, ils rencontrent de réelles difficultés à lister les demandes des lycéens et à faire attribuer des réalisations au conseil. De manière récurrente, ils s'interrogent sur la possibilité même de pouvoir représenter leurs camarades. Cette difficulté à entrer en relation avec le reste des lycéens nuit selon eux à la légitimité de leur parole. D'ailleurs, les adultes leur objectent qu'ils sont uniquement porteurs de revendications et d'analyses individuelles, nullement représentatives de l'ensemble des lycéens. L'absence de «parole collective » et d'« opinion commune » revient dans les propos des adultes les moins convaincus et leur permet de remettre en cause la légitimité des lycéens élus et, par là même, la validité des travaux du conseil. 
Cette incertitude quant au contenu de leur travail de délégué est accentuée par les conditions pratiques dans lesquels ils exercent leur mandat. Ainsi, les lycéens sont confrontés à au moins deux difficultés. D'une part, compte tenu de leurs appartenances à différentes classes, en particulier dans les LEGT et les lycées polyvalents, ils ne se connaissent pas. Il leur est, de ce fait, difficile de former un collectif et d'établir une collaboration efficace. D'autre part, compte tenu de la variation de leurs emplois du temps, ils ne disposent pas toujours de créneaux communs pour se rencontrer. Des solutions ont été trouvées dans certains établissements. Par exemple, dans un lycée, les délégués ont mis en place deux permanences d'une heure, les uns venant le lundi, les autres le jeudi. Dans un autre, ils se voient le jeudi pendant l'heure de vie de classe commune à l'ensemble du lycée. De ce fait, l'exercice de la fonction de délégué au conseil de la vie lycéenne nécessite à la fois une définition de ses contours et un aménagement de ses conditions d'exercice.

\section{Conclusion}

Ce cheminement des principes d'action au fonctionnement permet d'appréhender les différents processus qui structurent le fonctionnement du conseil de la vie lycéenne. En effet, si au départ sont affirmés des principes généraux, leur mise en œuvre est déjà fortement déterminée par l'organisation et la composition de cette instance. Ces éléments contribuent à configurer le format de l'échange avant même que ce dernier n'ait lieu. Dans les choix opérés par le ministère, le dialogue est censé s'établir entre un nombre équivalent de lycéens et d'acteurs de la communauté scolaire, porter sur les thématiques qui concernent directement le quotidien des lycéens et permettre, dans un cadre qui reste consultatif, aux délégués de disposer d'un pouvoir supplémentaire aux autres membres au travers de leur droit de vote. Le conseil de la vie lycéenne renforce le droit d'expression des lycéens qui semble peu opératoire dans les autres instances et rétablit au passage une forme d'égalité d'accès à la parole. Or, la configuration de l'échange est modifiée par un double processus de réception et de traduction qui se repère autant du côté des acteurs de la communauté scolaire invités à y siéger que de celui des lycéens.

Dans un premier temps, les lycéens s'inscrivent dans un processus d'évaluation des ressources dont ils disposent pour se faire entendre. Cette évaluation des conditions de possibilité du dialogue ne repose pas seulement sur leur perception ou leur expérience de la fonction de délégué de classe, mais sur celle, plus générale, du quotidien scolaire, dont celui de la classe. La crédibilité du conseil et, par là même, l'intérêt de s'y engager, repose sur la construction d'une confiance dans la possibilité d'un dialogue et dans celle d'être en présence d'interlocuteurs attentifs et loyaux. Dans un second temps, ce que met en évidence l'étude des thèmes abordés en réunion, les lycéens contribuent à la construction des objets 
discutables en apprenant à différencier ce dont ils aimeraient parler, de ce dont on aimerait qu'ils parlent et de ce dont ils peuvent effectivement parler. Ils opèrent progressivement une sélection des thèmes, sélection d'autant plus importante qu'ils ne disposent pas nécessairement des connaissances institutionnelles pour faire valoir certains points de vue et des compétences pratiques pour prendre la parole. À bien des égards, ils évaluent le coût de leur participation au débat. Si elle n'est pas adéquate, elle peut remettre en cause leur légitimité politique et les ramener à un rôle dont ils parviennent difficilement à sortir, celui de lycéen. Bien qu'ils soient appelés à endosser le rôle de citoyen ou celui d'usager, leur posture au sein du conseil, mais aussi la manière dont leur prise de parole ou leur action sont évaluées, témoignent d'une difficulté à agir et à être considérés autrement qu'en élève. Ainsi, le sens pratique qu'ils mobilisent et acquièrent au sein du conseil de la vie lycéenne pour s'inscrire dans l'échange prend clairement appui sur l'intériorisation d'un rôle qui n'est pas celui qu'ils sont censés jouer au sein de cette instance. Par conséquent, le conseil de la vie lycéenne apparaît, comme d'autres instances de participation ou comme d'autres démarches d'éducation à la citoyenneté, redéfini par la forme scolaire (Audigier, 2006).

Valérie Becquet, université de Cergy-Pontoise/IUFM de Versailles, CREF, université de Paris Ouest Nanterre La Défense.

\section{BIBLIOGRAPHIE}

AUdigIER François. L'éducation à la citoyenneté aux prises avec la forme scolaire, dans LENOIR Yves, Xypas Constantin, Jamet Christian (dir.). École et citoyenneté. Un défi multiculturel. Armand Colin, 2006.

BACQUÉ Marie-Hélène, Rey Henri et Sintomer Yves (dir.). Gestion de proximité et démocratie de proximité. Une perspective comparative. La Découverte, 2005 (Recherches).

BALlion Robert. La démocratie au lycée. ESF, 1998 (Pédagogies).

BALLION Robert. Le lycée, une cité à construire. Hachette éducation, 1993 (Questions d'éducation).

BARRÈre Anne. Sociologie des chefs d'établissement. Les managers de la République. PUF, 2006 (Éducation et formation).

BARRÈre Anne. Les lycéens au travail. PUF, 1997 (Pédagogie d'aujourd'hui).

BARRÈre Anne, MARTUCELli Danilo. La citoyenneté à l'école: vers la définition d'une problématique sociologique. Revue française de sociologie, 1998, vol. XXXIX, n 4, p. 651-671.

BECQUET Valérie. Dialoguer avec les jeunes: un engagement réciproque? Les conseils de la vie lycéenne, dans BECQUET Valérie et DE LINARES Chantal. Quand les jeunes s'engagent. Entre expérimentations et constructions identitaires. L'Harmattan, 2005a (Débats jeunesse).

BECQUET Valérie. Les conseils de la jeunesse à Paris. Atouts et difficultés d’un dispositif de démocratie participative. Paris: CAFI, 2005 b.

BECQUET Valérie, La presse lycéenne entre droits et infractions, dans HÉNAFF Gaël et MERLE Pierre (dir.). Le droit et l'école. De la règle aux pratiques. Rennes: PUR. 2003. 
BECQUET Valérie. Mise en place et fonctionnement des conseils de la vie lycéenne. Paris: Direction de l'enseignement scolaire, Ministère de la Jeunesse, de l'Éducation nationale et de la Recherche, 2002.

BLONDiAuX Loïc, MARCou Gérard et RANGEON François (dir.). La démocratie locale. Représentation, participation et espace public. PUF, 1999.

BLONDiAuX Loïc. La démocratie par le bas. Hermès, 2000, n² 26-27, p. 323-337.

BOUMARD Patrick. Le conseil de classe. Institution et citoyenneté. PUF, 1997 (L'éducateur).

CAllon Michel, Lascoumes Pierre et BARTHE Yannick (dir.). Agir dans un monde incertain, essai sur la démocratie technique. Seuil, 2001.

Cousin Olivier. L'effet établissement. Construction d'une problématique. Revue française de sociologie, 1993, vol. XXXIX, p. 395-419.

DUBET François. Les lycéens. Seuil, 1991.

DURAND-PRinborgne Claude. Élèves aujourd'hui, citoyens demain. Savoir, 1991, nº 1, p. 511-526.

DUTERCQ Yves. Administration de l'éducation: nouveau contexte, nouvelles perspectives. Revue française de pédagogie, 2000, n 130, p. 143-170.

FRANÇOIS Bastien et NEVEU Erik (dir.). Espaces publics mosaïques. Acteurs, arènes et rhétoriques des débats publics contemporains, Rennes: PUR, 1999 (Res Publica).

Guillaume Jean-Claude et Verdon Roseline. Pratiques citoyennes des lycéens dans et hors de létablissement. Ministère de l'Éducation nationale, de l'Enseignement supérieur et de la Recherche (Les dossiers Insertion, Éducation et Société), mai 2007, nº 184.

MERLE Pierre. L'élève humilié. L'école, un espace de non droit? Paris: PUF, 2005 (Éducation et formation).

MERLE Pierre. Les droits des élèves. Droits formels et quotidien scolaire des élèves dans l'institution éducative. Revue française de sociologie, 2001, vol. 42, n 1, p. 81-115.

MERLE Pierre. Les notes. Secrets de fabrication. PUF, 2007 (Éducation et Société).

Perrenoud Philippe. Métier délève et sens du travail scolaire. ESF, 1994 (Pédagogie recherche).

RAYOU Patrick. La Cité des lycéens. L'Harmattan, 1998 (Débats jeunesse).

ROBERT Francis. Enseigner le droit à lécole. ESF, 1999 (Pratiques et enjeux pédagogiques).

ROUDET Bernard. Conseil des délégués et droits des élèves. Quelle citoyenneté des lycéens pour quelle évolution du système scolaire?. Savoir, 1994, n³. SiROTA Régine. Le métier d'élève. Revue française de pédagogie, 1993, nº 104, p. 85-108.

SEMBEL Nicolas. Le travail scolaire. Nathan université, 2003 (Éducation 128).

SOUSSAN Michel. Vie scolaire: approche sociohistorique. Revue française de pédagogie, 1988, n 83 , p. 39-49. 
Claude Carpentier (coord.). L'école dans un monde en crise. Entre globalisation et héritages. L'Harmattan, 2008, (Éducation et société), 284 p. (25,65€).

es crises traversées par l'institution scolaire partout dans le monde se situent au point de convergence entre « modernité » dominée par le néolibéralisme et héritages historiques. La première a pour conséquence l'émergence d'un paradigme selon lequel l'éducation devient un produit soumis aux règles des échanges économiques et de la concurrence. Ce qui rend obsolète le principe d'une éducation considérée comme bien public au service de la réduction des inégalités.

Aboutissement du processus de développement du capitalisme, la crise actuelle de l'école témoigne également de l'héritage des bouleversements historiques liés à la décolonisation et aux nouveaux rapports «nord-sud » ainsi qu'à la conversion des sociétés " socialistes » au néolibéralisme. Dans les deux cas, la question des identités culturelles et ethniques est au cœur de la crise des institutions scolaires.

C'est dans ce contexte que les enseignants, acteurs privilégiés des crises de l'école, se trouvent confrontés à une expérience sociale traversée par les tensions, aussi bien en ce qui concerne leur identité professionnelle mise à rude épreuve par la transformation de l'institution qu'en ce qui concerne l'évolution des contenus d'enseignement eux-mêmes.

La coordination de l'ouvrage et la présentation des textes ont été assurées par des membres du CURSEP (Centre universitaire de recherche en sciences de l'éducation et en psychologie, équipe d'accueil 2089) : Claude Carpentier, directeur. Bruno Poucet, Teresa Longo, Philippe Monchaux, Yves Verneuil. 